\title{
The impact of mathematical competences and cognitive effort on the appearance of the framing effect ${ }^{1}$
}

\author{
Aleksander Kaczmarek ${ }^{2}$,Krzysztof Przybyszewski ${ }^{3}$, \\ Dorota Rutkowska ${ }^{4}$, Honorata Sosnowska ${ }^{5}$
}

\begin{abstract}
The aim of this paper is to check whether mathematical competences influence some manifestations of bounded rationality. A special example of bounded rationality called "framing effect" is dealt with to analyze empirically the thesis that mathematical competences and cognitive effort may reduce the framing effect. Two kinds of cognitive effort: probabilistic and deductive are analysed. Experiments were conducted using samples of Polish students, both mathematically and business oriented. As an example of a framing situation an example called "Asian disease", (the first analyzed and the most popular example of the framing effect), is considered. The thesis that a mathematical background may diminish the occurrence of the framing effect was partly confirmed.
\end{abstract}

Key words: bounded rationality, framing effect, experiment.

JEL codes: D910.

\section{Introduction}

The aim of this paper is to check whether mathematical competences impact some of the manifestations of bounded rationality. A special example of bounded rationality called "framing effect," is considered and an analysis is made empirically of the thesis that mathematical competences and cognitive effort may reduce the framing effect.

\footnotetext{
${ }^{1}$ Article received 5 August 2017, accepted 30 March 2018. The research underlaying this paper was financed partly by KAE SGH (grant KAE/S/35/13).

${ }^{2}$ Copernicus Science Center, Wybrzeże Kościuszkowskie 20, 00-390 Warsaw, Poland.

${ }^{3}$ Kozminski University, Center for Economic Psychology and Decision Sciences, ul. Jagiellońska 57/59, 03-301 Warsaw, Poland.

${ }^{4}$ Warsaw University, Faculty of Psychology, Department of Cognitive Psychology, ul. Stawki 5/7, 00-183 Warsaw, Poland.

${ }^{5}$ Warsaw School of Economics, Collegium of Economic Analysis, Department of Mathematics and Mathematical Economics, al. Niepodległości 162, 02-554 Warsaw, Poland, honorata@sgh.waw.pl.
} 
Two experiments were conducted using students of Warsaw universities who possessed different mathematical competences. Differences of mathematical levels in any scale were not measured but they were diagnosed in considering the students' majors. Firstly, because matriculation in mathematics was not obligatory at that time. Secondly, students who studied mathematics at various levels during their studies were considered and this fact significantly influenced their mathematical competences depending on the type of studies. Other characteristics were randomized because groups were based on university lists such as alphabetical order or university number. In the case of students with high mathematical competences these were based on an interest in subject of mathematics and quantitative methods. Experiments were conducted in similar circumstances such as a period of time, a part of a day, a part of a semester. Students displayed similarity in their life style and approach to studies. Mathematical competences were the only significant difference. Other characteristics may play a role in case of some individuals, but they constitute a small percentage as in any randomized group.

In the first experiment students of Warsaw School of Economics with high and low mathematical backgrounds were compared. Both groups answered questions on Asian disease and a part of them were stimulated by probabilistic cognitive effort. Results showed that mathematical competences only partly diminished the framing effect (in case of loss). The conclusion was drawn that our respondents did not differ sufficiently in terms of their level of mathematical competence and we conducted the second experiment In this experiment students of mathematics and physics were compared with students of management. The results confirmed the results of the first experiment (that mathematical competences diminished the framing effect in case of loss). The impact of the mathematical background was greater than in the first experiment.

The paper is organized as follows. In Section 1 bounded rationality as the foundation of the framing effect is presented. The Asian disease experiment is explained in section 2. Hypotheses are presented in Section 3. The first experiment (Sosnowska, 2013) is analyzed in Section 4, the second experiment (Kaczmarek, 2015) in Section 5. The paper ends with conclusions.

\section{Bounded rationality}

Making a decision under risk, especially in the domain of social affairs, is quite often a trade-off between being economically rational and socially acceptable. A decision-maker may employ rational models of analysis, i.e., expected value, but in the real world such a 'ruthless' approach to the decisions may not find wide support amongst the public. Arguably most real decision makers are perfectly aware of this. Beach and Lipshitz (1996) describe it as an "open situation" with some unpredictable social risks involved as opposed to the "closed" ones 
with all the consequences well-specified and not going beyond the matter to be decided upon. For instance a decision maker who is to decide whether to treat patient A or B with a scarce medication should focus on the chances for recovery, however he/she would probably take into account some moral considerations (about who is to be saved, etc.). The concept that explains this theoretical standpoint is Simon's bounded rationality. In an objectively defined world agents could be perfectly, i.e., economically, rational, yet in the real world the understanding of the dilemma to be resolved is by all means a subjective one, based upon the decision-maker's own personal goals, not necessarily consistent with the expected value maximizing principle. The above concerns both micro and macroeconomics (Kowalski, 2002). Being aware of the consequences that go beyond the decision scenario, or focusing on non-economic aspects of the dilemma may lead to tagging the decision as moral, ethical, religious, or personal vs. impersonal. As will be described later the tag attached to the decision scenario may lead to major changes in the nature of the decision-making process: the motives and goals are different, the amount of effort varies and finally, different decision rules are employed.

The framing effect may be treated as a part of dual brain processing. Let us study a short framework for framing studies.

The problem of different risk preferences in the domains of gains and losses was addressed by Markowitz (1952). For large outcomes people are risk averse in the domain of gains and risk seeking in the domain of losses, which was later named as the "framing effect". However Markowitz proposed that for the small outcomes the reverse framing effect is expected - risk seeking in small gains and risk aversion in small losses. Obviously for the "Asian disease scenario" when massive (non-financial) outcomes are presented, the Markowitz theory and the Prospect theory suggest the same pattern of risk preferences.

The contemporary approach to this problem, called the "framing effect" has its beginning in Tversky and Kahneman's (1981) research.

The framing effect may be described as the breaking of the invariance principle caused by putting a decision-maker in the domain of gain or loss. Logically the risk preference should be stable, however in the domain of loss people tend to seek risk, while in the domain of gain they chose the certain option.

The framing effect is, superficially, a well-documented bias in risky decisions (see Kühberger, 1998; Kühberger, Schulte-Mecklenbeck, \& Perner, 1999). Regardless of this little is known about the nature of the processes that lead to its occurrence. It is especially not clear whether the effect is produced by the lack of or excess of thought. Some of the cognitive biases and errors are clearly categorized as produced by shallow thoughts while others result from deep, yet erroneous thinking. The results of the framing studies, however, do not allow such a strong statement to be made. The amount of cognitive effort involved in the processing is said to reduce the effect (Guo, Trueblood, \& Diederich, 2017), although some empirical results demonstrate the opposite - thoughtful 
processing generates the effect (Gonzales, Dana, Kosino, \& Justa, 2005; Igou \& Bless, 2007; Svenson \& Benson, 1993).

\section{Asian disease as a classical scenario for framing studies}

Since 1981 the framing effects (Tversky \& Kahneman, 1981) have been studied extensively, most often in connection with the "Asian disease" problem: the scenario in which a deadly disease endangers the lives of 600 inhabitants of a certain town. The task is to choose between two alternative rescue programmes, either certain or risky, which are described (framed) either positively or negatively, but equal in their expected value.

Positively framed subjects choose between:

(A) saving 200 people for sure and (B) saving 600 people with a one-third probability and a two-thirds probability that no people will be saved.

Negatively framed subjects choose between:

(A') certain death of 400 people and (B') a one third probability that nobody will die and a two-thirds probability that all 600 people will die.

The framing effect shows itself in violation of the invariance principle, i.e., choosing a risky gamble ( $\left.B^{\prime}\right)$ over a certain thing $\left(A^{\prime}\right)$, when the descriptors are negative (i.e., in loss domain, 78\% chose $B^{\prime}$ ) and sure option (A) over a gamble (B) when the descriptors are positive (i.e., in gain domain, $72 \%$ chose A).

The description of this bias is found in the Prospect Theory (Kahneman \& Tversky, 1979) which states that people have an s-shaped value-function, concave for gains (which makes people risk-averse) and convex for losses (which makes people risk-seeking). Each prospect is evaluated (i.e., moved up or down around the reference point) as gain or loss and choices are made correspondingly.

The effect seems not to be equal in size and strength under different conditions. There can be found some systematic individual differences in susceptibility to the framed choices also. Most of the studies were aimed at finding the most efficient methods for de-biasing the choices by increasing the amount of cognitive effort invested in the decision making process. This approach is based upon the assumption that the effect stems from reflexive or automatic processes and it can be overcome by thorough, motivated thought.

The first stream of research focuses on various techniques of increasing the amount of thought - by making the participants accountable for the results of the decision, typically by either informing them that they would be asked to write a justification or actually writing it (Takemura, 1994). Takemura's study supported this method, however the results obtained by Sieck and Yates (1997) show that only getting the subjects to write the account removes the effect, which may mean that it is not only the motivation but also a 're-framing' of the scenario that reduces the effect. Contrary to those findings, in the 
study by Igou and Bless (2007), with the manipulation of the importance of the choice ('serious' vs. 'pilot' study tags) the effect disappeared in participants held accountable in a study tagged as a 'pilot' study, but was present in the one representing a 'serious' condition. The possible reason for the inconsistency of the results may be the spontaneous emergence of different goals the decisionmakers may pursue in the process: logical correctness or creating a compelling narrative explaining the choice they made.

This idea was tested in a set of studies where the goal was presented to the participants by tagging the problem as 'medical' (i.e., pertaining to the mor$\mathrm{al} /$ ethical domain) or 'statistical' (clearly aimed at logical correctness). In the study by Igou and Bless (2007) where the participants were to solve either $a$ statistical problem or a medical problem the framing effect was obtained only in the group of participants solving the medical problem. The authors claim that the framing effect is produced by constructive information processing in the course of which affectively vivid but non-diagnostic cues (e.g., words as 'die' and 'save') start to have an impact on the choices, possibly because they lead to moral considerations about the consequences of the decisions. In the 'statistical' condition the diagnostic data are contained in the numerical values which naturally draws decision-makers' attention to the calculations and expected value of the options.

Another stream of studies is based upon seeking individuals' mental traits and capabilities that prevent them from making biased decisions. The qualities of the mind suspected as playing a role in the decision making are either the natural need to make reflective and difficult choices or mathematical literacy. Indeed in the study by Simon, Fagley and Halleran (2004) people with a high level of the need for cognition and high self-evaluation of their mathematical skills displayed no framing effect in the Asian disease scenario, while in the participants with a low need for cognition, the framing effect was obtained regardless of their mathematical skills. Frederick (2005) assumed that both logical and mathematical skills, as well as the motivation to suppress intuitive answers would influence decision making when the decision problems call for the rules of normative reasoning. The motivation to suppress first intuitions was measured by the Cognitive Reflection Test. Frederick (2005) observed that only the participants with low CRT scores more frequently chose the sure option in the domain of gain and the lottery in the domain of loss. People with high results in CRT were, however, more prone to take risk in the gain frame, avoid it in the loss frame and follow the expected value principle in their choices.

Risk choices such as in the case of the occurrence of a framing effect may be connected with some additional reasons. An influence of higher education was analyzed by Fan (2017) on a Chinese example. Experiments described by Sparks and Ledgerwood (2017) show the dependence of risk decisions on some additional activities (in this case - the sequential framing effect). The research dealt with differences in education (mathematical or business) and the impact 
of additional activities (probabilistic or deductive incentives). The results do not cover the above mentioned but are based on the same method of seeking conditions which cause a framing effect.

\section{Hypotheses}

In the experiments presented above, a more formal way of introduction (e.g., the use of the word "statistical" instead of "medical") may reduce the framing effect. There may be a correlation between the mathematical competences of respondents and the occurrence of the framing effect because there is a conviction that people with high mathematical competences reason logically and therefore they are more resistant to the framing effect. Two experiments where respondents were divided into two groups - more and less mathematically oriented. Both groups completed a questionnaire with questions on the choice of programme in the Asian disease problem, half of them in the domain of gain, and half - domain (of what - this does not make sense).

In these experiments an attempt is made to confirm the following hypotheses.

H1: Mathematical competences cause a lack of the framing effect.

H2: Probabilistic cognitive effort causes a lack of the framing effect.

H3: Deductive cognitive effort causes a lack of the framing effect.

H4: High mathematical competences cause probabilistic equivalence of programmes A and B (A' and $\left.B^{\prime}\right)$ to be observed more frequently than in case where such competences are low.

The experiments are described in the next sections.

\section{First experiment - probabilistic incentives}

The first experiment was conducted by Sosnowska in 2013, with two groups of students of the Warsaw School of Economics (SGH).

The first group consisted of first-year BA students. They only had basic mathematical competences but most of them passed extended mathematics as part of the matriculation exam (the group will be denoted as Nmat). They had just started their studies and learnt only a bit of mathematics. They also had not attended lectures on quantitative methods in economics as yet. The students were further randomly divided into two groups, one where a probabilistic incentive to cognitive effort was applied and second without such an incentive. A simple exercise about the probability of gathering special mushrooms, which implied intuitions connected with expected value, was used as the manipulation. These students did not know probability calculus. Groups will be denoted NmatPro 
- with, and NmatNpro without the manipulation. In both groups subgroups which operate in the gain domain (NmatProG, NmatNproG) and loss domain (NmatProL, NmatNproL) were created.

The second group of respondents consisted of second and third-year students specializing in quantitative methods at the Warsaw School of Economics. All of them had many lectures on advanced mathematics including the probability theory. Their mathematical competences based on their university mathematical education were significantly higher than those of the first group because they had much more experience in mathematics. This group of students will be denoted as Mat. They were divided into two groups, with (MatPro) and without (MatNpro) probabilistic incentive to cognitive effort. The experiment in the group with the incentive was conducted as part of an examination on probabilistic calculus, where students had to solve a task on expected value. The examination and this exercise played the role of the incentive. In both groups, subgroups were identified one operating in the domain of gain (MatProG, MatNproG) and the other operating in the domain of loss (MatProL, MatNproL). The numbers of respondents in each subgroup are presented in Table 1.

It is shown in Table 1 that there was at least approximately 20 students in each group.

Table 1. Number of respondents in each subgroup

\begin{tabular}{|l|c|}
\hline \multicolumn{1}{|c|}{ Subgroup } & Number of respondents \\
\hline NmatProG & 18 \\
\hline NmatProL & 44 \\
\hline NmatNproG & 37 \\
\hline NmatNproL & 42 \\
\hline MatProG & 26 \\
\hline MatProL & 32 \\
\hline MatNproG & 28 \\
\hline MatNproL & 32 \\
\hline
\end{tabular}

Source: Own calculations.

In Table 2 the occurrence of the framing effect in groups NmatPro, NmatNpro, Matpro and MatNpro is presented. In the following tables the sum of percentages may not be equal to $100 \%$ because some respondents noted an equivalence of programmes or gave irrelevant answers. In the statistical analysis the critical value is 3.84 with $\alpha=0.05$.

Table 2 shows that there is no unambiguous answer to hypothesis H1, H2.

In Table 3 respondents are divided into groups with (Mat $G=$ MatProG + + MatNproG, MatL $=$ MatProL + Mat NproL $)$ and without $(N m a t G=N m a t P r o G+$ 
Table 2. Occurrence of the framing effect

\begin{tabular}{|c|c|c|c|}
\hline Group & $\begin{array}{c}\text { Chosen } \\
\text { A or A' }(\%)\end{array}$ & $\begin{array}{c}\text { Chosen } \\
\text { B or B' (\%) }\end{array}$ & Framing effect \\
\hline NmatProG & 55.0 & 33.0 & \multirow{2}{*}{$\begin{array}{l}\text { Chi square 3.60. It is impossible to check } \\
\text { unambiguously the framing effect }\end{array}$} \\
\hline NmatProL & 34.1 & 63.6 & \\
\hline NmatNproG & 51.3 & 48.6 & \multirow{2}{*}{$\begin{array}{c}\text { Chi square 1.40. It is impossible to check } \\
\text { unambiguously the framing effect }\end{array}$} \\
\hline NmatNproL & 38.1 & 61.9 & \\
\hline MatProG & 57.7 & 19.2 & \multirow{2}{*}{ Chi square 4.44 . There is a framing effect } \\
\hline MatProL & 37.5 & 46.9 & \\
\hline MatNproG & 42.8 & 46.4 & \multirow{2}{*}{$\begin{array}{l}\text { Chi square 0.56. It is impossible to check } \\
\text { unambiguously the framing effect }\end{array}$} \\
\hline MatNproL & 56.2 & 43.8 & \\
\hline
\end{tabular}

Source: Own calculations.

+ NmatNproG, Nmat $L=$ NmatProL + NmatNproL) mathematical competences. The occurrence of the framing effect is studied (the last column).

Table 3. Comparison of respondents with and without mathematical competences

\begin{tabular}{|c|c|c|c|}
\hline Group & $\begin{array}{c}\text { Chosen } \\
\text { A or } A^{\prime}(\%)\end{array}$ & $\begin{array}{c}\text { Chosen } \\
\text { B or B' (\%) }\end{array}$ & Framing effect \\
\hline MatG & 36.5 & 63.5 & \multirow{2}{*}{$\begin{array}{c}\text { Chi square 1.88. It is impossible to check } \\
\text { unambiguously the framing effect }\end{array}$} \\
\hline MatL & 40.0 & 46.7 & \\
\hline Nmat $G$ & 52.7 & 43.6 & \multirow{2}{*}{ Chi square 4.43 . There is a framing effect } \\
\hline NmatL & 36.5 & 63.5 & \\
\hline
\end{tabular}

Source: Own calculations.

It is shown in Table 3 that hypothesis $\mathrm{H} 1$ is confirmed. More precisely it is shown in Figure 1.

Results described in Table 3 show occurrence of the framing effect presented in Figure 1.

In Table 4 respondents are divided into groups with (ProG $=$ MatProG + + Nmat ProG, ProL = MatProL + NmatProL $)$ and without $($ NproG $=$ MatNproG + + NmatProG, NproL = MatNproL + NmatNproL) probabilistic incentives.

It is shown in Table 4 that hypothesis $\mathrm{H} 2$ is not confirmed. This fact is presented more precisely in Figure 2.

Occurrence of the framing effect is presented in the Figure 2.

In Table 5 the percentage of respondents who noted the equivalence of programmes is presented. 
Table 4. Comparison of respondents with and without probabilistic incentive

\begin{tabular}{|c|c|c|c|}
\hline Group & $\begin{array}{c}\text { Chosen } \\
\text { A or A' }(\%)\end{array}$ & $\begin{array}{c}\text { Chosen } \\
\text { B or B' (\%) }\end{array}$ & Framing effect \\
\hline ProG & 56.8 & 25.0 & \multirow{2}{*}{ Chi square 9.14. There is a framing effect } \\
\hline ProL & 36.0 & 57.3 & \\
\hline NproG & 47.7 & 47.7 & \multirow{2}{*}{$\begin{array}{c}\text { Chi square 0.30. It is impossible to check } \\
\text { framing effect unambigously }\end{array}$} \\
\hline NproL & 45.9 & 54.0 & \\
\hline
\end{tabular}

Source: Own calculations.

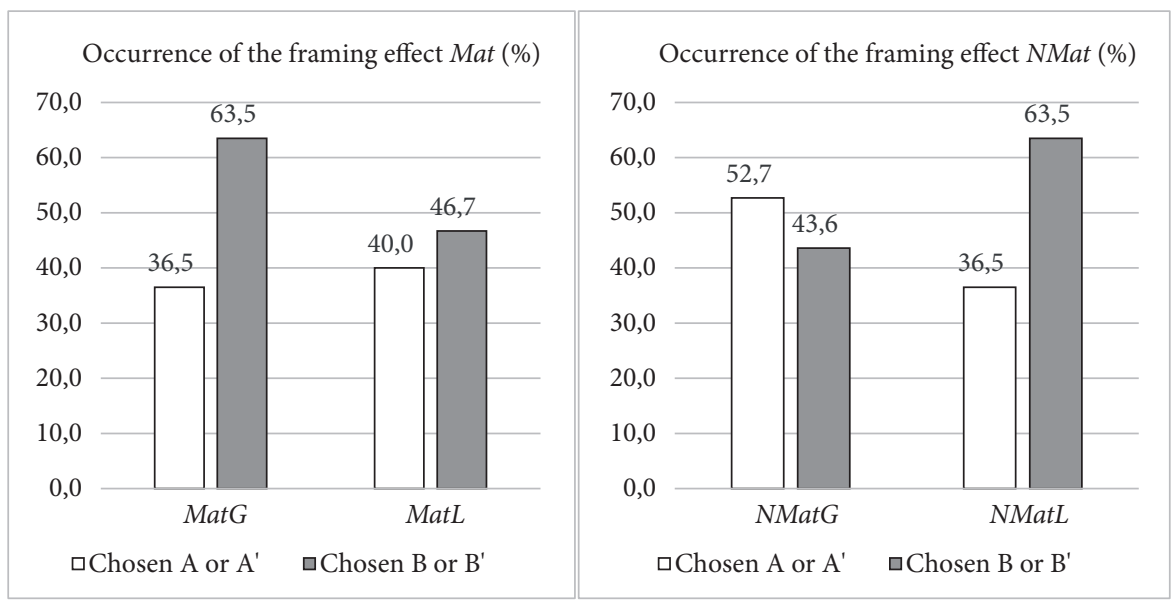

Figure 1. Comparison of respondents with and without mathematical competences

Source: Own calculations.

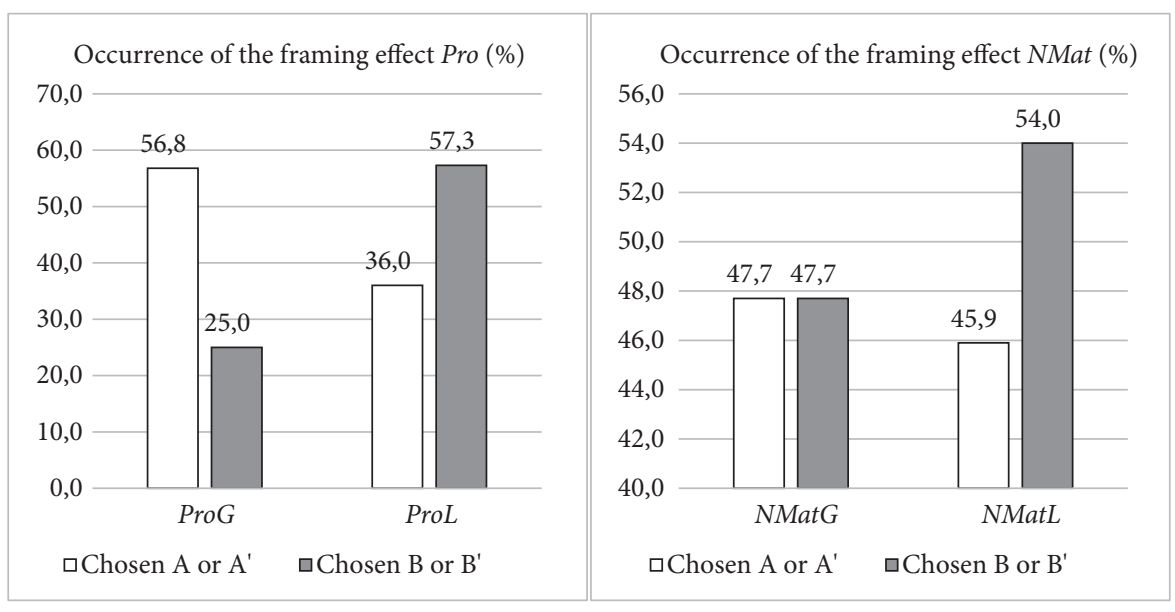

Figure 2. Comparison of respondents with and without probabilistic incentive Source: Own calculations. 
Table 5. Equivalence of programs

\begin{tabular}{|l|c|}
\hline \multicolumn{1}{|c|}{ Group } & $\begin{array}{c}\text { Respondents who } \\
\text { noted equivalence of } \\
\text { programmes (\%) }\end{array}$ \\
\hline NmatPro & 1.6 \\
\hline NmatNpro & 1.3 \\
\hline MatPro & 31.0 \\
\hline MatNpro & 13.3 \\
\hline
\end{tabular}

Source: Own calculations.

It is shown in Table 5 that hypothesis H4 is confirmed. Probabilistic incentives may make this effect stronger.

\section{Second experiment - deductive incentives}

The second experiment was conducted by Kaczmarek in 2015 using two groups of students of Polish universities. This time students differed a lot in their mathematical competences. The first group consisted of students of management at Kozminski University (denoted as Nmat). They knew only elementary mathematics and only participated in an introductory lecture on the application of mathematics in economics. Their mathematical competences were low, comparable to the British $\mathrm{O}$-level examination. Two of the authors have experience in teaching in business universities and they have knowledge about their students' mathematical competence in comparison with students of mathematics or physics. They did not know the probability theory. They were divided into two groups, one where some deductive incentives were introduced and a second without such incentives. A logical riddle was used as manipulation Groups will be denoted NmatDed - with, and NmatNded - without the manipulation. In both groups subgroups operating in the domain of gain (NmatDedG, NmatNdedG) and the domain of loss (NmatDedL, NmatNdedL) were identified.

The second group of students consisted of students of mathematics and physics at Warsaw University and Warsaw University of Technology. Their mathematical competences were high, they completed many courses on advanced mathematics. They were divided into two groups, one where some deductive incentives were introduced and a second - without such incentives. A geometrical exercise was used as an incentive. The groups will be denoted MatDed - with the incentive, and MatNded - without the incentive. In both groups subgroups operating in the domain of gain (MatDedG, MatNdedG), and in the domain of loss (MatDedL, MatNdedL) were identified. The number of respondents in each subgroup is presented in Table 6. 
Table 6. Number of respondents in each subgroup

\begin{tabular}{|l|c|}
\hline \multicolumn{1}{|c|}{ Subgroup } & Number of respondents \\
\hline NmatDedG & 22 \\
\hline NmatDedL & 19 \\
\hline NmatNdedG & 20 \\
\hline NmatNdedL & 17 \\
\hline MatDedG & 21 \\
\hline MatDedL & 21 \\
\hline MatNdedG & 29 \\
\hline MatNdedL & 21 \\
\hline
\end{tabular}

Source: Own calculations.

It is shown in Table 6 that each subgroup is consisted of approximately 20 students.

In Table 7 the occurrence of the framing effect is presented. In the statistical analysis the critical value is 3.84 with $\alpha=0.05$.

Table 7. Occurrence of framing effect

\begin{tabular}{|c|c|c|c|}
\hline Group & $\begin{array}{c}\text { Chosen } \\
\text { A or } A^{\prime}(\%)\end{array}$ & $\begin{array}{c}\text { Chosen } \\
\text { B or B' (\%) }\end{array}$ & Framing effect \\
\hline MatDedG & 57 & 43 & \multirow{2}{*}{ No framing effect } \\
\hline MatDedL & 57 & 43 & \\
\hline MatNdedG & 69 & 31 & \multirow{2}{*}{$\begin{array}{l}\text { Chi square } 3.41 \text {. } \\
\text { It is impossible to check the framing ef- } \\
\text { fect unambiguously }\end{array}$} \\
\hline MatNdedL & 43 & 57 & \\
\hline NmatDedG & 54 & 46 & \multirow{2}{*}{$\begin{array}{l}\text { Chi square } 0.22 \text {. } \\
\text { It is impossible to check the framing ef- } \\
\text { fect unambiguosusly }\end{array}$} \\
\hline NmatDedL & 47 & 53 & \\
\hline NmatNdedG & 65 & 35 & \multirow{2}{*}{$\begin{array}{c}\text { Chi square } 6.36 . \\
\text { Framing effect }\end{array}$} \\
\hline NmatNdedL & 23 & 77 & \\
\hline
\end{tabular}

Source: Own calculations.

It is shown in Table 7 that hypotheses $\mathrm{H} 1$ and $\mathrm{H} 3$ are partly confirmed.

In Table 8 respondents are divided into groups with $($ Mat $G=\operatorname{MatDed} G+$ + MatNdedG, MatL = MatDedL + MatNdedL $)$ and without $(N m a t G=N m a t D e d G+$ + NmatNdedG, NmatL $=$ NmatDedL + NmatNdedL) mathematical competences. The occurrence of the framing effect is studied (the last column) and the 
conformity of numbers of respondents choosing programmes with expected numbers (the fourth column). Expected numbers are $50 \%$ and $50 \%$ because the programmes are equivalent.

Table 8. Comparison of respondents with and without mathematical competences

\begin{tabular}{|c|c|c|c|}
\hline Group & $\begin{array}{c}\text { Chosen } \\
\text { A or A' }(\%)\end{array}$ & $\begin{array}{c}\text { Chosen } \\
\text { B or B' (\%) }\end{array}$ & Framing effect \\
\hline MatG & 64 & 36 & \multirow{2}{*}{$\begin{array}{l}\text { Chi square } 1.84 \text {. } \\
\text { It is impossible to check unambiguously } \\
\text { the framing effect }\end{array}$} \\
\hline MatL & 50 & 50 & \\
\hline NmatG & 60 & 40 & \multirow{2}{*}{$\begin{array}{l}\text { Chi square } 4.04 \text {. } \\
\text { Weak framing effect }\end{array}$} \\
\hline NmatL & 36 & 64 & \\
\hline
\end{tabular}

Source: Own calculations.

It is shown in Table 8 that hypothesis $\mathrm{H} 1$ is confirmed. It is presented more precisely in Figure 3.
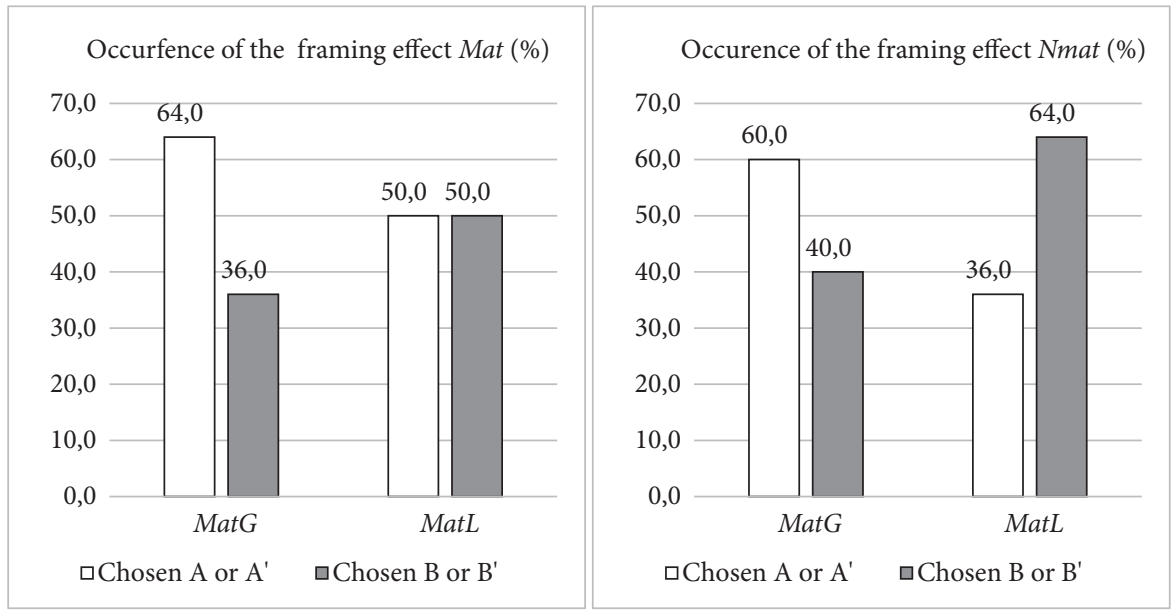

Figure 3. Comparison of respondents with and without mathematical competences

Source: Own calculations.

Occurrence of the framing effect is presented in Figure 3.

In Table 9 respondents are divided into groups with $(\operatorname{Ded} G=M a t D e d G+$ + NmatDedG, DedL $=$ MaDedL + NmatDedL $)$ and without $(N d e d G=M a t N d e d G+$ + NmatdedG, NdedL $=$ MatNdedL + NmatNdedL) probabilistic incentives. 
Table 9. Comparison of respondents with and without probabilistic incentive

\begin{tabular}{|l|c|c|c|}
\hline \multicolumn{1}{|c|}{ Group } & $\begin{array}{c}\text { Chosen } \\
\text { A or A' (\%) }\end{array}$ & $\begin{array}{c}\text { Chosen } \\
\text { B or B' (\%) }\end{array}$ & Framing effect \\
\hline DedG & 56 & 44 & $\begin{array}{c}\text { Chi square } 0.1 . \\
\text { It is impossible to check unambiguously } \\
\text { the framing effect }\end{array}$ \\
\hline DedL & 53 & 47 & $\begin{array}{c}\text { Chi square } 9.46 . \\
\text { Framing effect }\end{array}$ \\
\hline$N d e d G$ & 67 & 33 & Fnnn \\
\hline$N d e d L$ & 34 & 66 & \\
\hline
\end{tabular}

Source: Own calculations.

It follows from Table 9 that hypothesis $\mathrm{H} 3$ is confirmed. It is showed more precisely in Figure 4.

Occurrence of the framing effect is presented in Figure 3.

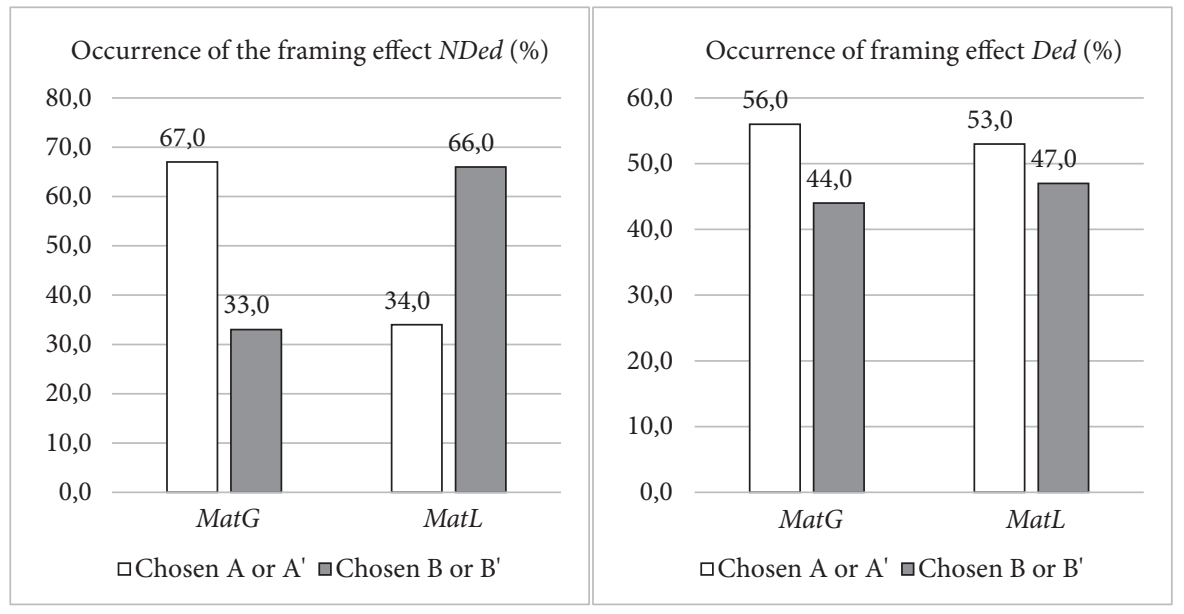

Figure 4. Comparison of respondents with and without probabilistic incentive Source: Own calculations.

Table 10. Equivalence of programmes

\begin{tabular}{|l|c|}
\hline \multicolumn{1}{|c|}{ Group } & $\begin{array}{c}\text { Respondents who } \\
\text { noted equivalence of } \\
\text { programmes (\%) }\end{array}$ \\
\hline MatDed & 21 \\
\hline MatNded & 26 \\
\hline NmatDed & 0 \\
\hline NmatNded & 3 \\
\hline
\end{tabular}

Source: Own calculations. 
In Table 10 the percentage of respondents who noted the equivalence of programmes is presented.

It follows from Table 10 that hypothesis $\mathrm{H} 4$ is confirmed. Impact of deductive incentives is not observed.

\section{Conclusions}

The research shows that hypothesis $\mathrm{H} 1$ on the lack of a framing effect in the presence of mathematical competences was partly confirmed. There are situations where mathematical competences may cause decisions to be more rational. Hypothesis $\mathrm{H} 2$ on the impact of a probabilistic incentive on the rationality of decision-making over risk was not confirmed. Contrary to the last observation hypothesis $\mathrm{H} 3$ about the impact of a deductive incentive was confirmed. It may be interpreted that in a situation with deductive incentives the Kahneman brain II starts to work (Kahneman, 2011). Indeed in a think-aloud experiment Maule (1989) demonstrated that people can spontaneously reframe the "Asian disease" problem (elaborating it as both gain and loss) which removed the bias. (Arkes 1991) obtained similar results using a direct reframing instruction. Framing effects also appeared less frequently in the within-subjects design (LeBoeuf \& Shafir, 2003). All of the above induce deductive reasoning.

Probabilistic reasoning is difficult to use without hints and probably has a different cognitive structure. Mathematical competences allow for an observation that the programmes are equivalent in their expected value, yet they still differ in complexity and social context. Hypothesis $\mathrm{H} 4$ was confirmed. Probabilistic or deductive incentives make this observation more frequent. To summarize there is some impact of mathematical competences and deductive reasoning on the occurrence of the framing effect but they do not eliminate it. The question of whether mathematical competences make reasoning more rational requires analysis based on more examples. The study is limited to analyses of students with a lack of financial incentives. The limitations of the study indicate possible directions of future research.

The first possible further research is to take essential financial incentives into consideration. The future study has to be conducted over a paid portal with an appropriate sum of money as remuneration. The second is comparing students of mathematics or mathematicians with a control group consisting of people unrelated to institutions of higher education.

The possible development of research should follow the results that confirm H4. It may be argued that not only the motivation (and hence the cognitive effort) and competences (and hence the capability to solve the problem) are responsible for the occurrence or diminishing of the framing effect. The idea of the aims or goals behind the thinking processes seems to be a prom- 
ising explanation, i.e., the strenuous and competent thought, yet oriented at a normatively correct solution would remove the effect, while when aimed at 'socially desirable' solutions, it may be responsible for the occurrence of the effect.

\section{References}

Arkes, H. R. (1991). Costs and benefits of judgement errors: implications for debiasing. Psychological Bulletin, 110(3), 486-498.

Beach, L. R., \& Lipshitz, R. (1996). Why a new perspective on decision making is needed. In L. R. Beach (ed.), Decision making in the workplace: a unified perspective. NJ, Erlbaum.

Fan, W. (2017). Education and decision-making: an experimental study on the framing effect in China. Frontiers in Psychology, https://doi.org./10.3389/fpsyg.2017.00744

Frederick, S. (2005). Cognitive reflection and decision making. Journal of Economic Perspectives, 19(4), 25-42.

Gonzales, C., Dana, J., Koshimo, H., \& Just, M. (2005). The framing effect and risky decisions: examining cognitive functions with fMRI. Journal of Economic Psychology, 26(1), 1-20.

Guo, L., Trueblood, J. S., \& Diederich A. (2017). Thinking fast increases framing effects in risky decision making. Psychological Sciences, 28(4), 530-543.

Igou, R., \& Bless, H. (2007). On undesirable consequences of thinking. Framing effect as a function of substantive processing. Journal of Behavioral Decision Making, 20(2), 125-142.

Kaczmarek, A. (2015). Wpłw wykształcenia matematycznego oraz dedukcyjnego wysitku poznawczego na wystapienie efectu sformułowania w problemie choroby azjatyckiej (Impact of mathematical education and cognitive deductive effort on appearance of framing effect in problem of Asian disease), BA thesis, Warsaw School of Economics.

Kahneman, D. (2011). Thinking Fast and Slow. New York: Farrar, Strauss \& Giroux.

Kahneman, D., \& Tversky, A. (1979). Prospect theory: an analysis of decision under risk. Econometrica, 47(2), 263-292.

Kowalski, T. (2002). The Simonian bounded rationality hypothesis and the expectation formation mechanism. Poznań University of Economics Review, 2(1), 5-24.

Kühberger, A. (1998). The influence of framing on risky decisions: a meta-analysis. Organizational Behavior and Human Decision Processes, 75(1), 23-55.

Kühberger, A., Schulte-Meckleenbeck, M., \& Perner, J. (1999). The effect of framing reflection probability and payoff on risk preference in choice tasks. Organizational Behavior and Human Decision Processes, 78(3), 204-231.

LeBoeuf, R. A, \& Shafir, E. (2003). Deep thoughts and shallow frames: on the susceptibility to framing effects. Behavioral Decision Making, 16(3), 77-92.

Markowitz, H. (1952). The utility of wealth. Journal of Political Economy, 60, 151-158. Maule, A. J. (1989). Positive and negative decision frames: a verbal protocol analysis of the Asian disease problem of Kahneman and Tversky. In O. Svenson, \& H. Montgomery (Eds.), Process and structure in human decision making (pp. 163-180). Chichester: John Wiley and Sons. 
Sieck, W., \& Yates, Y.F. (1997). Exposition effects on decision making. Choice and confidence in choice. Organizational Behavior and Human Decision Processes, 70(3), 207-219.

Simon, A. F., Fagley, N. S., \& Halleran, J. G. (2004). Decision framing. Moderating effects of individual differences and cognitive processing. Journal of Behavior Decision Making, 17(2), 77-93.

Sosnowska, H. (2013) Wplyw kompetencji matematycznych i probabilistycznego wysitku poznawczego na występowanie efektu sformułowania (Impact of mathematical competences and probabilistic cognitive effort on appearence of framing efffect), report on grant KAE/S/35/13, Collegium of Economic Analysis, Warsaw School of Economics, Warsaw.

Sparks, J., \& Ledgerwood, A. (2017). When good is stickier than bad: understanding gain/loss asymmetries in sequential framing effects. Journal of Experimental Psychology, http://dx.doi.org/10.1037/xge0000311

Svenson, O., \& Benson, L. III (1993). Framing and time pressure in decision making. In O. Svenson, A. J. Maule (Eds.), Time pressure and stress in human judgment and decision making (pp. 133-144). New York: Plenum.

Takemura, K. (1994). Influence of elaboration on the framing of decisions. The Journal of Psychology, 128(10), 33-39.

Tversky, A., Kahneman, D. (1981). A framing of decisions and psychology of choice. Science, 211, 453-458. 